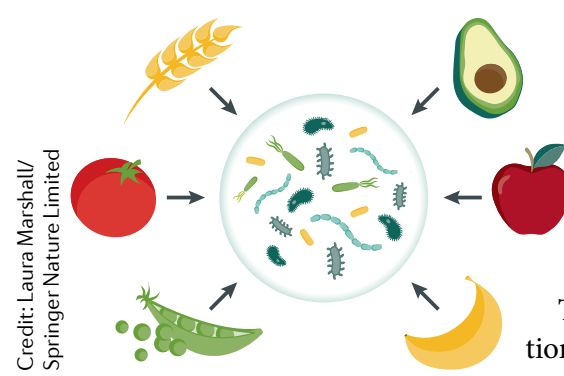

the presence of B. cellulosilyticus and avoided competing for fibre.

To study the competition between Bacteroides species, the team used artificial food particles: fluorescently labelled glass beads each coated with a fibre-derived glycan. These particles were introduced into the intestines of gnotobiotic mice containing different combinations of Bacteroides strains and then retrieved, in effect acting as biosensors for fibre digestion.

"These findings reveal the complexity that underlies the overlapping capacities of different gut microbes to process

To study the competition between

Bacteroides species, the team used artificial food particles

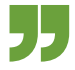

a fungal genus
abundant in
PDAC tumours
promotes
cancer
progression
dietary fibre," says Michael Patnode, the first author of the study. The group now hopes to apply their new approaches to investigate the effects of fibres on intact human gut microbiota.

Jordan Hindson

ORIGINAL ARTICLE Patnode, M. L. et al. Interspecies competition impacts targeted manipulation of human gut bacteria by fiber-derived glycans. Cell 179, 59-73 (2019)

in response to fungal pathogens. In PDAC mouse models, deletion of $\mathrm{Mbl}$ delayed tumour progression, and Malassezia-associated tumour progression was lost. Recombinant C3a, which is generated by complement activation, was found to accelerate the proliferation of tumour cells in vitro and tumour growth in vivo, and mice deficient in $\mathrm{C} 3$ or $\mathrm{C} 3$ a receptor were protected from tumour progression. Thus, the authors hypothesize that oncogenic Kras-induced inflammation results in fungal dysbiosis, which promotes cancer progression via the MBL-induced C3 cascade.

Going forward, the team hopes to identify fungi that could act as potential therapeutics against pancreatic cancer. "We are also interested in determining whether fungal signatures can be used as biomarkers of disease or predictors of outcomes," say Miller and Saxena.

Jordan Hindson

ORIGINAL ARTICLE Aykut, B. et al. The fungal mycobiome promotes pancreatic oncogenesis via activation of MBL. Nature 574, 264-267 (2019)

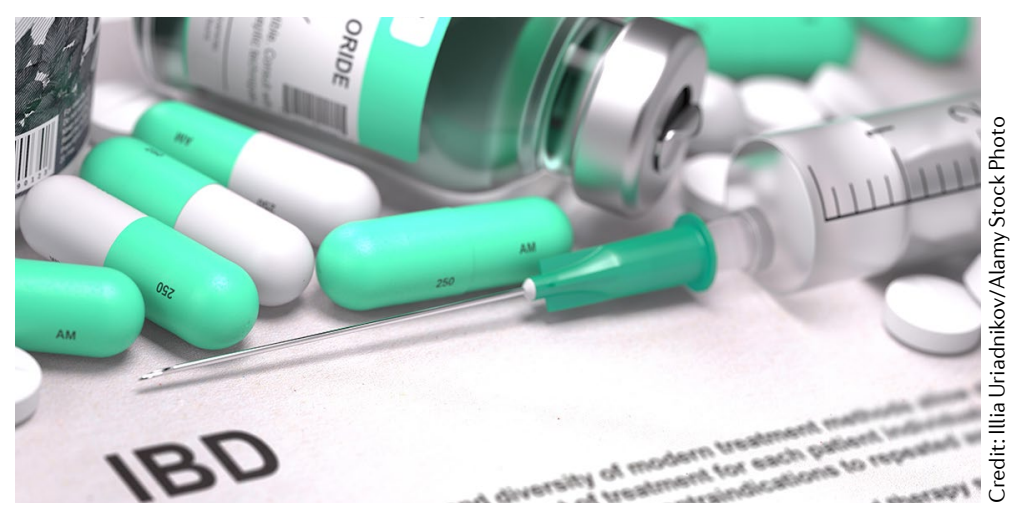

IBD

\section{New trials in ulcerative colitis therapies}

The results of two international phase III trials in ulcerative colitis have been published in the New England Journal of Medicine. The data show that, in patients with moderateto-severe ulcerative colitis, ustekinumab is effective as an induction and maintenance therapy and vedolizumab is superior to adalimumab in a head-to-head comparison.

In the UNIFI trial, the investigators aimed to demonstrate the efficacy of IL-12 and IL-23 blockade in ulcerative colitis using the monoclonal antibody ustekinumab. "This drug was already shown to be effective and approved for Crohn's disease more than 3 years ago," explains Bruce Sands, the corresponding author on both the new studies. "There was a strong mechanistic rationale for its efficacy in ulcerative colitis as well, given that genomic studies in IBD demonstrate a protective allele in the IL-23 receptor gene."

A total of 961 patients with moderate-to-severe ulcerative colitis were recruited to the UNIFI trial, which examined ustekinumab versus placebo as an 8-week induction therapy and then as a 44-week maintenance therapy in patients that responded. "The UNIFI study was the first example of a randomized controlled trial that examined "histoendoscopic healing' as an endpoint," says Sands. "By combining features of established definitions of endoscopic improvement with a definition of histologic improvement that allows for histologic findings of neutrophil infiltration in $<5 \%$ of crypts, no crypt destruction, and no erosions, ulcerations, or granulation tissue, histo-endoscopic healing was defined, and in fact achieved, in patients treated with ustekinumab." Ustekinumab was found to be significantly more effective than placebo for inducing and maintaining remission based on a range of outcomes, and the safety profile was similar to placebo. "Importantly, it was effective in both anti-TNF-naive and anti-TNF-failure patients," says Sands.

The VARSITY trial was notable for being the first example of a blinded, headto-head trial of two biologic agents, vedolizumab and the anti-TNF agent adalimumab. "It was widely assumed that TNF inhibitors would be more effective as well as more rapid in onset of effect than vedolizumab, a gut-selective anti-integrin antibody. Therefore, we set out to compare them in a direct fashion," explains Sands.

A total of 769 patients with moderate-to-severe ulcerative colitis were randomly assigned to receive one of the biologic agents. "The surprising finding was that vedolizumab was more effective than adalimumab in achieving clinical remission at week 52, which was the primary endpoint," reports Sands. "This effect was most notable in the TNF-inhibitor-naive patients, who comprised $80 \%$ of the patients." Although numerical rates of steroid-free clinical remission at week 52 were higher in the adalimumab arm than the vedolizumab arm, the difference from vedolizumab was not significantly different, statistically. Both agents had similar safety profiles.

The investigators think their novel head-to-head approach could have immediate implications for the positioning of these agents in treating patients with ulcerative colitis. "It will be exciting to see other head-to-head studies in IBD, and some are already ongoing. Over time, these will inform how best to sequence treatments to achieve maximum efficacy," concludes Sands.

lain Dickson

ORIGINAL ARTICLES Sands, B. E. et al. Ustekinumab as induction and maintenance therapy for ulcerative colitis. N. Engl.J. Med. 381, 1201-1214 (2019)| Sands, B. E. et al. Vedolizumab versus Adalimumab for

Moderate-to-Severe Ulcerative Colitis. N. Engl.J. Med. 381, 1215-1226 (2019) 